1

2

3

4

5

6

7 8

11

12

13

14

15

16

17

18

19

20

21

22

23

24

25 ORCID:

26 Gregory Dachner: 0000-0002-9198-330X

27 Trenton Wirth: 0000-0002-2510-8603

28 Emily Richmond: 0000-0001-8667-0969

29 William Warren: 0000-0003-4843-2315
30

31

32

33

34

35

\section{The visual coupling between neighbors explains 'flocking' in human crowds}

Gregory C. Dachner, Trenton D. Wirth, Emily Richmond, and William H. Warren*

Department of Cognitive, Linguistic, and Psychological Sciences

Brown University, Providence, RI 02912

${ }^{*}$ Corresponding Author

Email: Bill Warren@brown.edu 


\section{Abstract}

37 Patterns of collective motion or 'flocking' in birds, fish schools, and human crowds are believed to emerge 38 from local interactions between individuals. Most models of collective motion attribute these interactions

39 to hypothetical rules or forces, often inspired by physical systems, and described from an overhead view.

40 We develop a visual model of human flocking from an embedded view, based on optical variables that

41 actually govern pedestrian interactions. Specifically, people control their walking speed and direction by

42 canceling the average optical expansion and angular velocity of their neighbors, weighted by visual

43 occlusion. We test the model by simulating data from experiments with virtual crowds and real human

44 'swarms'. The visual model outperforms our previous overhead model and explains basic properties of

45 physics-inspired models: 'repulsion' forces reduce to canceling optical expansion, 'attraction' forces to canceling optical contraction, and 'alignment' to canceling the combination of expansion/contraction and angular velocity. Critically, the neighborhood of interaction follows from Euclid's Law of perspective and the geometry of occlusion. We conclude that the local interactions underlying human flocking are a species. 


\section{Introduction}

52 Human crowds exhibit patterns of collective motion in many public settings, from train stations and shopping plazas to - sometimes catastrophically - mass events (Helbing, Buzna, Johansson, \& Werner, 2005; Ngai, Burkle, Hsu, \& Hsu, 2009). Similar patterns of coordinated motion are observed in bird flocks, fish schools, and animal herds, suggesting that diverse systems may obey common principles of selforganization (Couzin \& Krause, 2003; Sumpter, 2010). It is generally believed that these global 'flocking' patterns emerge from local interactions between individuals (Couzin \& Krause, 2003; Sumpter, 2010; Vicsek \& Zafeiris, 2012). The crux of the problem thus lies in understanding the nature of the local interactions.

Most models of collective motion ascribe these local interactions to hypothetical rules or forces, often inspired by physical systems (Giardina, 2008; Schellinck \& White, 2011). Such descriptive models including our own (Rio, Dachner, \& Warren, 2018) - assume an overhead view of the positions and velocities of all individuals in space. But humans and animals are immersed in a group and coupled by sensory information. Here we develop a visual model of collective motion that explains local interactions in terms of optical variables, from the viewpoint of an embedded agent. Not only does this explanatory model outperform our previous descriptive model, but basic properties of interaction turn out to be natural consequences of the laws of optics.

Understanding the local interactions involves, first, identifying the rules of engagement that govern how an individual responds to a neighbor, and second, characterizing the neighborhood of interaction over which the influences of multiple neighbors are combined. Classical "zonal" models (Couzin, Krause, James, Ruxton, \& Franks, 2002; Huth \& Wissel, 1992; Reynolds, 1987) assume three local rules or forces in concentric zones: (i) repulsion from neighbors in a near zone to avoid collisions, (ii) alignment with the velocity of neighbors in an intermediate zone to generate common motion, and (iii) attraction to neighbors in a far zone to ensure group cohesion. Neighbor influences are combined by averaging within a zone (Couzin et al., 2002; Huth \& Wissel, 1992; Reynolds, 1987), often weighted by neighbor distance (Cucker \& Smale, 2007; Grégoire, Chaté, \& Tu, 2003; Mogilner, Edelstein-Keshet, Bent, \& Spiros, 2003), and obey the superposition principle, according to which the response to a group is the linear combination of independent responses to each neighbor. An alignment rule by itself is theoretically sufficient to generate collective motion (Leonard et al., 2012; Vicsek, Czirók, Ben-Jacob, Cohen, \& Shochet, 1995), as is the combination of attraction and repulsion (Romanczuk, Couzin, \& Schimansky-Geier, 2009). Among pedestrian models (Chraibi, Tordeux, Schadschneider, \& Seyfried, 2018), the prominent Social Force model (Chen, Treiber, Kanagaraj, \& Li, 2018; Helbing \& Molnár, 1995; Hoogendoorn \& Bovy, 2003) is predicated on attraction and repulsion forces and has convenient mathematical properties (Köster, Treml, \& Gödel, 2013). The model successfully simulates key crowd scenarios (Boltes, Zhang, Tordeux, Schadschneider, \& Seyfried, 2018; Chraibi et al., 2018) and can generate collective motion under certain conditions (Helbing, Farkas, \& Vicsek, 2000; Helbing, Molnár, Farkas, \& Bolay, 2001), but does not produce realistic human trajectories (Pelechano, Allbeck, \& Badler, 2007) or generalize to novel situations without re-parameterization (Campanella, Hoogendoorn, \& Daamen, 2009; Chen et al., 2018).

The strength of such physics-inspired models is that they capture generic properties of collective motion, but the metaphor does not transfer smoothly to biological agents (Schellinck \& White, 2011; Weitz et al., 2012). Because different sets of rules can produce similar global patterns (Vicsek \& Zafeiris, 2012; Weitz et al., 2012), some researchers have turned to a 'bottom-up' approach that deploys experimental studies of behavioral interactions to infer the local rules (Calovi et al., 2018; Gautrais et al., 2012; Katz, Tunstrøm, loannou, Huepe, \& Couzin, 2011; Moussaid et al., 2009; Sumpter, Mann, \& Perna, 2012; Warren \& Fajen, 2008; Zienkiewicz, Barton, Porfiri, \& Di Bernardo, 2015). We further argue that a successful bottom-up model must be grounded in the sensory information that actually governs these interactions.

Initially, limits on the field of view and sensory range were introduced to constrain models of local interactions (Huth \& Wissel, 1992; Partridge \& Pitcher, 1980; Pita, Collignon, Halloy, \& Fernández-Juricic, 2016). An analysis of fish schools discovered that a neighborhood based on visibility accounted for individual responses better than zonal or topological neighborhoods (Strandburg-Peshkin et al., 2013).

100

101 Local interactions also strongly depend on the optical information that controls locomotion (Gibson, 1979; 
102

103

104

105

106

107

108

109

110

111

112

113

114

115

116

117

118

119

120

121

122

123

124

125

126

127

128

129

130

(a)

131

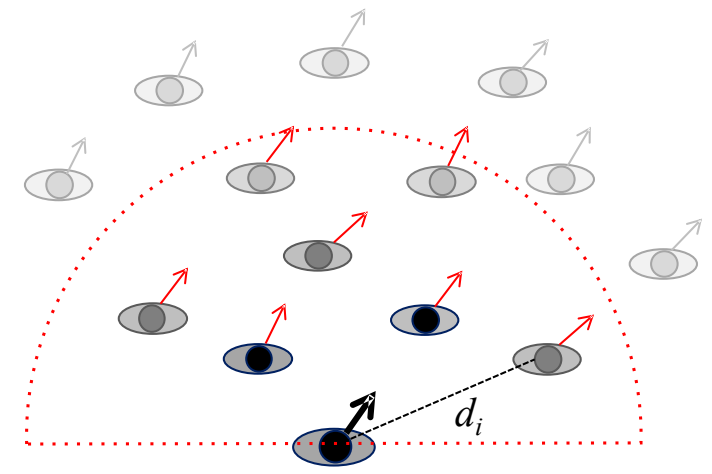
based simulations. with distance, going to zero at about 4-5m (Rio et al., 2018).

Pepping \& Grealy, 2007; Warren, 1998). This insight was taken up by 'vision-based' (Dutra, Marques, Cavalcante-Neto, Vidal, \& Pettré, 2017; Ondrej, Pettré, Olivier, \& Donikian, 2010) and 'cognitive heuristic' (Bailo, Carrillo, \& Degond, 2018; Moussaïd, Helbing, \& Theraulaz, 2011) models, which were based on properties such as time-to-closest-approach, distance-at-closest-approach, or an interaction force related to time-to-contact (Karamouzas, Skinner, \& Guy, 2014); however, the underlying optical variables were not specified. Recently, a minimal theoretical approach has been developed (Bastien \& Romanczuk, 2020), which starts with simple visual directions as input and studies the emergent behavior in agent-

We take a complementary, experiment-driven approach called 'behavioral dynamics' (Warren, 2006; Warren \& Fajen, 2008), in which control is based on higher-order optical variables that animals are known to exploit(Frost \& Sun, 2004; Srinivasan, 1998; Warren, Kay, Zosh, Duchon, \& Sahuc, 2001). To model collective motion from the bottom up, we began with experiments on following in pedestrian dyads (Dachner \& Warren, 2014; Rio, Rhea, \& Warren, 2014). The results revealed that humans obey an alignment rule: a follower tends to match the walking direction (heading) and speed of the leader. We then mapped the neighborhood of interaction by asking participants to walk with a crowd in virtual reality, which allowed us to precisely control the movements of their virtual neighbors. The results showed that pedestrians average the heading directions and speeds of neighbors within the field of view, while analysis of naturalistic data from a human 'swarm' found that neighbor influence decays exponentially

These findings led to Rio, Dachner \& Warren's (2018) descriptive behavioral model of collective motion (Figure 1a; see SI). In brief, steering (angular acceleration) is controlled by canceling the difference between one's current heading and the weighted average of neighbor headings. Speed (linear acceleration) is similarly controlled by canceling the difference between current speed and the weighted average of neighbor speeds. The weights decay exponentially with neighbor distance within a $180^{\circ}$ field of view, yielding a semi-circular 'soft metric' neighborhood that generates robust collective motion in simulation (Cucker \& Smale, 2007; Warren \& Dachner, 2018). The model was fit to our data on pedestrian dyads, with the decay rate taken from the human swarm, and successfully predicted individual trajectories in virtual crowd experiments and real crowd data.

Figure 1. Overhead and embedded models of collective motion. (a) Overhead behavioral model: a pedestrian (bottom) matches the average heading direction of all neighbors in a $180^{\circ}$ neighborhood. Neighbor weights (gray level) decay exponentially with distance $d_{i}$ and go to zero at 4-5m (dotted red curve). (b) Embedded visual model: a pedestrian (bottom) cancels the average angular velocity and optical expansion/contraction of all visible neighbors. Neighbor influence (gray level) decreases due to Euclid's law of perspective and is proportional to visibility (shaded areas indicate occluded regions). 
141

142

143

144

145

146

147

148

149

150

151

152

153

154

155

156

157

158

159

160

161

162

163

164

165

166

167

(a)

\section{Results}

\section{Behavioral results}

\section{The neighborhood of interaction is not fixed}

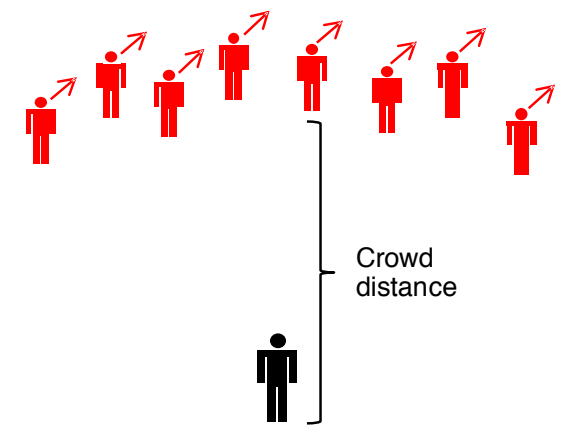

The behavioral model describes an empirical relation between physical variables - the velocity of an individual and the positions and velocities of neighbors. Like its predecessors, it assumes an overhead view, relies on metaphorical forces, and does not explain the local interactions. To address these issues, we developed a visual model predicated on the optical variables that are known to control pedestrian following (Figure 1b). This new model explains local interactions and the form of the neighborhood as resulting from the laws of optics and the geometry of visual occlusion.

According to the behavioral model, the neighborhood of interaction has a fixed radius, such that the influence of neighbors decays to zero at 4-5m (Rio et al., 2018). We tested this limit in a simple experiment by manipulating the distance to a virtual crowd and perturbing heading direction. To elicit collective-motion responses, participants $(n=12)$ were instructed to "walk with the group of virtual humans" and "treat them as if they were real people." A row of virtual humans $(2,4$, or 8$)$ was presented in a wireless head-mounted display, and its initial distance was manipulated $(1.8,3.0,4.0,6.0$ or $8.0 \mathrm{~m})$ (Figure 2a). On each trial, they appeared with their backs to the participant, began walking forward (1.0 $\mathrm{m} / \mathrm{s}$ ), and after $5 \mathrm{~s}$ all turned by $10^{\circ}$ (left or right) in the same direction and continued walking for another $7 \mathrm{~s}$. There was thus no occlusion. We used a head tracker to record the participant's position and computed a time series of heading for each trial. The dependent measure was the participant's final heading, the average heading direction during the last $2 \mathrm{~s}$ of each trial (left/right turns were collapsed).

To our surprise, we observed a very gradual decay in neighbor influence over a long distance (Figure 2b). The participant's response decreased from a maximum final heading $\left(M=9.55^{\circ}\right)$ at $1.8 \mathrm{~m}$ to just half that value $\left(M=5.16^{\circ}\right)$ at $8 \mathrm{~m},\left(F(4,44)=14.93, p<0.001, \eta_{G}^{2}=0.290\right)$. Linear extrapolation implies that the response would decay to zero around $15 \mathrm{~m}(y=-0.722 x+10.8, r(14)=-0.95)$. There was no effect of crowd size on final heading $\left(F(2,22)=0.77, p=0.476, \eta_{G}^{2}=0.010\right)$ and no interaction $(F(8,88)=0.83$ $\left.p=0.575, \eta_{G}^{2}=0.033\right)$, consistent with weighted averaging of neighbors.

(b)

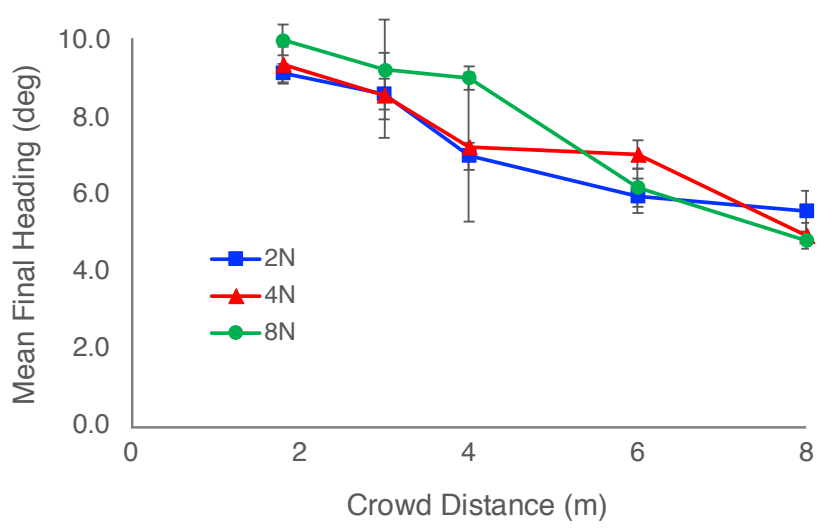

Figure 2. First experiment: Gradual decay to nearest neighbors. (a) Schematic of virtual crowd, illustrating a heading perturbation of all neighbors to the right. The distance between the participant (bottom) and the row of neighbors (top), and the number of neighbors, were manipulated. (b) Results: Mean final heading as a function of crowd distance. Curves represent the number of neighbors in the crowd; error bars represent \pm SE. 
175

176

177

178

179

180

181

182

183

184

185

186

187

188

189

190

191

192

193

194

195

196

197

198

The results clearly show that, contrary to a neighborhood with a fixed radius of $4-5 \mathrm{~m}$, pedestrians interact with neighbors at much greater distances - when they are not occluded. These findings imply that there may be two decay rates with distance: a gradual decay to the nearest neighbors, and a more rapid decay within a crowd. We tested this 'double-decay' hypothesis in the next experiment.

\section{The double-decay hypothesis}

To test two decay processes with distance, we asked each participant $(n=10)$ to walk with a virtual crowd of 12 neighbors, positioned in three rows (Figure 3a). We replicated the decay to the nearest neighbors by manipulating the distance of the near row $(2,4$, or $6 \mathrm{~m}$ from the participant), and probed the decay within the crowd by selectively perturbing the near, middle, or far row (always $2 \mathrm{~m}$ apart). At the perturbation, all neighbors in one row turned by $10^{\circ}$ in the same direction. Otherwise, the procedure was the same as before.

Figure $3 \mathrm{~b}$ plots mean final heading as a function of distance to the perturbed row, where each curve represents a crowd distance $(2,4$, or $6 \mathrm{~m})$. Two decay rates are immediately apparent. First, the heading response decreases with the overall distance of the crowd, $\left(F(2,18)=8.59, p=0.002, \eta_{G}^{2}=0.127\right)$; in particular, the response to perturbations of the near row (leftmost point of each curve) decays gradually with distance (simple effect test, $F(2,18)=12.196, p<0.001)$, replicating the previous experiment. Linear extrapolation suggests that responses would go to zero around $10 \mathrm{~m}(y=-.44 x+4.5, r(2)=-.97)$. Second, in each curve the response decreases rapidly within the crowd, $(F(2,18)=35.64, p<0.001$, $\eta_{G}^{2}=0.596$ ), steeply from the near row to the middle row (Bonferroni-corrected $t(9)=8.822, \mathrm{p}<0.001$ ) and the far row $(t(9)=7.718, p<0.001)$, which approaches zero. This finding suggests that partial occlusion by near neighbors significantly weakened the influence of middle neighbors, and far neighbors were almost completely occluded. In a separate experiment (Dachner \& Warren, 2019), we confirmed that visual occlusion reduces heading responses by manipulating the amount of occlusion.

(a)

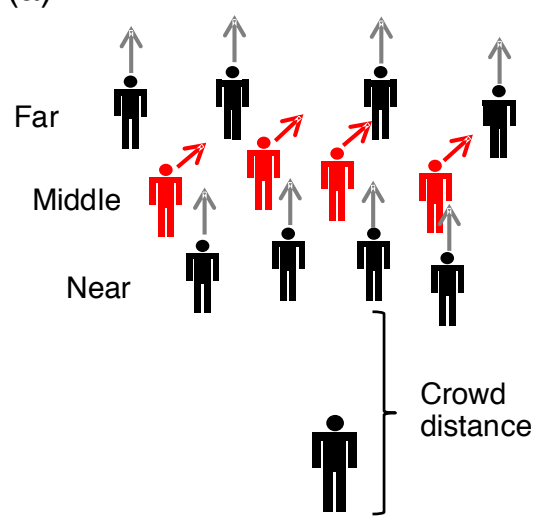

(b)

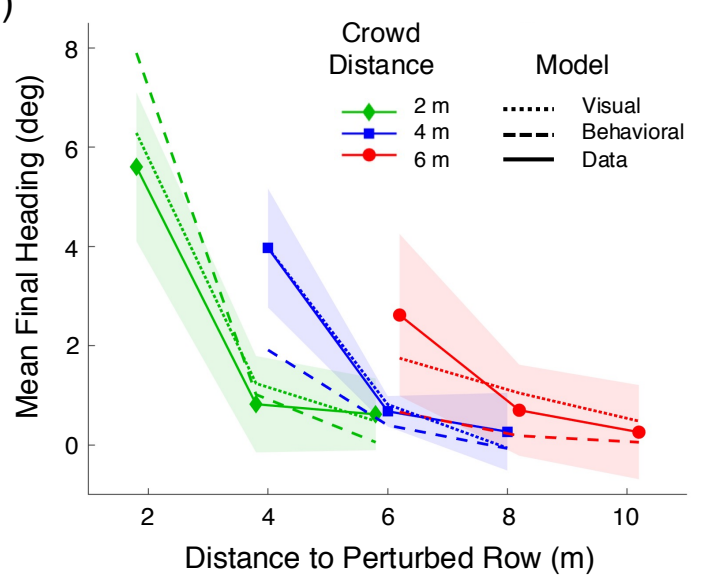

Figure 3. Second experiment: Test of the double-decay hypothesis. (a) Schematic of virtual crowd, illustrating a heading perturbation of one row to the right. The distance to the crowd (near row), and the perturbed row (near, middle, far), were manipulated. (b) Results: Mean final heading as a function of distance to the perturbed row, for each crowd distance (curves). Solid curves correspond to the human data, dotted curves to the visual model, and dashed curves to the behavioral model with the double-decay function. Shaded regions represent $95 \%$ confidence intervals for the human data; because the models are not intended to reproduce gait oscillations, their variable error is small and is not represented in the graph. 
The evidence thus reveals that the neighborhood of interaction results from two decay rates. But what are the underlying processes that produce them? We propose that the gradual decay to nearest (unoccluded) neighbors follows from Euclid's law of perspective, while the more rapid decay within the crowd is due to the added effect of occlusion. These findings led to a new visual model.

\section{Visual model}

To build a visual model of collective motion from the bottom up, we begin with the visual coupling between a pedestrian and a single neighbor (Bai \& Warren, 2019; Dachner \& Warren, 2017; Rio et al., 2014).

Heading control. Consider a pedestrian who is walking with a neighbor directly ahead of them (eccentricity $=0^{\circ}$ ) (see Figure 4). If the neighbor turns left, this generates a leftward angular velocity in the pedestrian's field of view (Figure 4a); the pedestrian could cancel the angular velocity by steering left (and vice versa for a right turn). On the other hand, if the neighbor is on the pedestrian's right (eccentricity $=90^{\circ}$ ) and turns left, this generates an optical expansion in the field of view (Figure 4b); in this case, the pedestrian could cancel the expansion by steering left. Conversely, a right turn by the neighbor generates an optical contraction, which could be canceled by steering right.

(a)

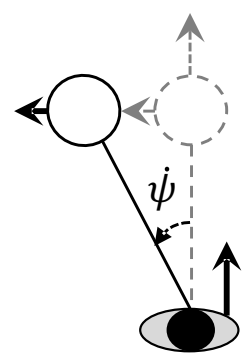

(c)

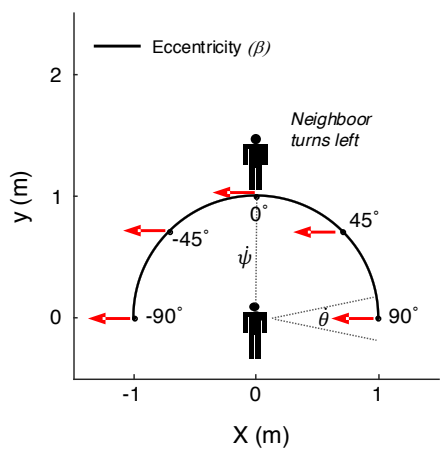

(b)

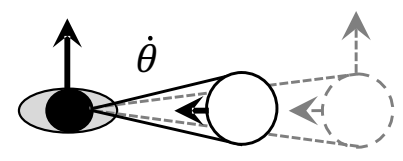

(d)

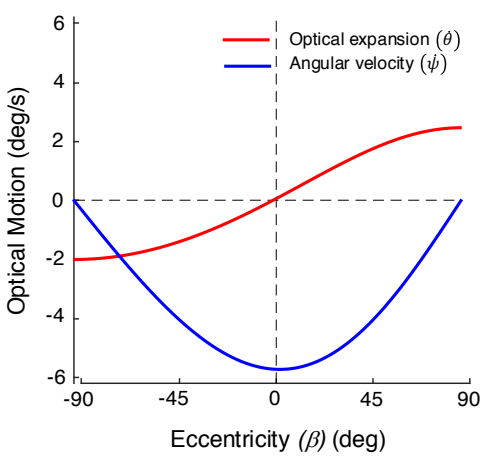

Figure 4. Visual information for the control of heading (walking direction). (a) If a neighbor (open circle) directly ahead of the pedestrian (filled oval) turns left, this creates a leftward angular velocity $(\dot{\psi})$ in the pedestrian's field of view, which the pedestrian can cancel by turning left. (b) If a neighbor on the right side of the pedestrian turns left, this creates an optical expansion $(\dot{\theta})$ in the field of view, which the pedestrian can also cancel by turning left. (c) The optical motions thus depend on the neighbor's eccentricity ( $\beta$, black curve), illustrated by a top-down view of a neighbor at a distance of $1 \mathrm{~m}$, turning $90^{\circ}$ to the left. (d) The resulting optical motion (deg/s) as a function of eccentricity $(\beta)$ : Angular velocity (blue curve) is a cosine function of eccentricity, with zero-crossings at $\beta= \pm 90^{\circ}$ and a minimum at $\beta=0^{\circ}$ (leftward motion). In contrast, expansion rate (red curve) is a sine function of eccentricity, with a zero-crossing at $\beta=0^{\circ}$, a maximum at $\beta=90^{\circ}$ (expansion), and a minimum at $\beta=-90^{\circ}$ (contraction). Note that if the neighbor turns right, these curves are flipped about the $x$-axis. 
These two optical variables thus trade off as a function of the neighbor's eccentricity $(\beta)$ (Figure 4c,d): If the neighbor turns left, their angular velocity $(\dot{\psi})$ is a cosine function of eccentricity with a minimum (leftward velocity) at $\beta=0^{\circ}$. In contrast, their rate of expansion $(\dot{\theta})$ is a sine function with a maximum at $\beta=90^{\circ}$ (the minimum at $\beta=-90^{\circ}$ corresponds to contraction). For a right turn, the sine and cosine functions simply flip about the x-axis. Critically, both optical velocities $(\dot{\psi}, \dot{\theta})$ decrease with neighbor distance in accordance with Euclid's law of perspective, which states that the visual angle subtended by an object, or a motion, diminishes with distance from the observer (specifically, as $\tan ^{-1}(1 / d)$ ).

The visual coupling for heading $(\phi)$ can thus be formalized as a second-order control law,

$$
\ddot{\phi}_{p}=-c_{1}\left(\cos \beta_{i}\right) \dot{\psi}_{\iota}+c_{2}\left(\sin \beta_{i}\right) \dot{\theta}_{i}
$$

in which pedestrian $p$ steers (angular acceleration $\ddot{\phi})$ so as to cancel the combined angular velocity $(\dot{\psi})$ and expansion rate $(\dot{\theta})$ of neighbor $i$. The dependence of these variables on eccentricity $(\beta)$ acts as a filter so the pedestrian is only influenced by components of angular velocity and expansion/contraction that specify a turning neighbor. The free parameters $\left(c_{1}=14.38, c_{2}=59.71\right)$ were fit to our previous data on pedestrian following in dyads (Dachner \& Warren, 2017) and held constant.

Speed control. The control of walking speed is complementary to the control of heading (see Figure 5). If a neighbor directly ahead $\left(\beta=0^{\circ}\right)$ slows down, this generates an optical expansion in the pedestrian's field of view (Figure 5a); and if the neighbor speeds up, it generates an optical contraction. But if a neighbor to the pedestrian's right slows down $\left(\beta=90^{\circ}\right)$, this generates a negative (leftward) angular velocity (Figure $3 \mathrm{~b}$ ), and vice versa. The two optical variables again trade off as a function of eccentricity, but with the opposite the sine and cosine functions (Figure $5 \mathrm{c}, \mathrm{d}$ ). Once again, by Euclid's law, the optical velocities decrease with neighbor distance.

The visual coupling for radial speed $(\dot{r})$ is thus based on the same two optical variables as Equation 1, but the sine and cosine functions are reversed:

$$
\ddot{r}_{p}=-c_{3}\left(\sin \beta_{i}\right) \dot{\psi}_{i}-c_{4}\left(\cos \beta_{i}\right) \dot{\theta}_{i}
$$

Pedestrian $p$ thus linearly accelerates or decelerates $(\ddot{r})$ so as to cancel the combined angular velocity $(\dot{\psi})$ and expansion rate $(\dot{\theta})$ of the neighbor $i$, but the pedestrian is now influenced only by combinations that specify a change in neighbor speed. The free parameters $\left(c_{3}=0.18, c_{4}=0.72\right)$ were fit to our data on following in pedestrian dyads (Dachner \& Warren, 2017) and held fixed. To account for variation in neighbor size, the relative rate of expansion $(\dot{\theta} / \theta)$ can be substituted for the expansion rate $(\dot{\theta})$, thereby normalizing it by the neighbor's visual angle $(\theta)$ (Bai \& Warren, 2019; Wagner, 1982). 
(a)

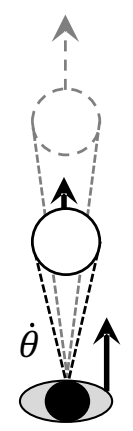

(c)

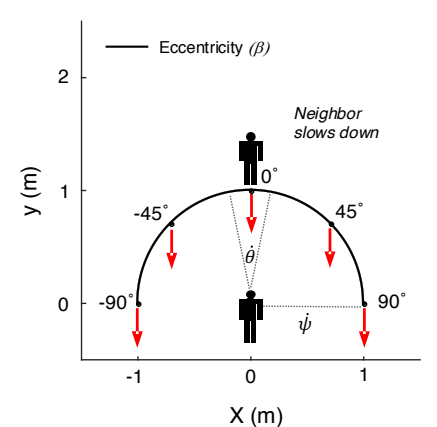

(b)

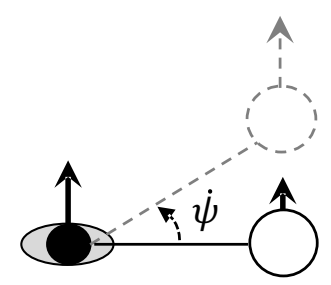

(d)

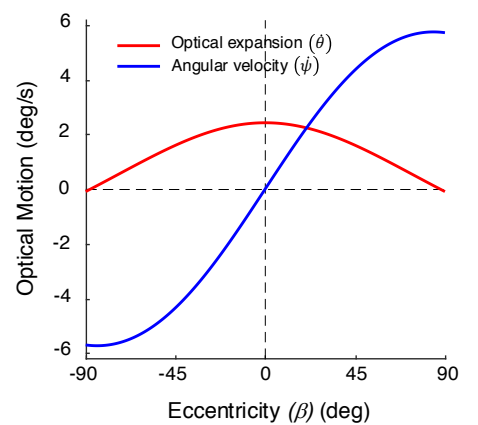

Figure 5. Visual information for the control of walking speed. (a) If a neighbor directly ahead (open circle) slows down, this creates an optical expansion $(\dot{\theta})$ in the pedestrian's field of view, which the pedestrian (filled oval) can cancel by decelerating. (b) If a neighbor to the pedestrian's right slows down, this creates an angular velocity $(\dot{\psi})$ in the field of view, which can also be cancelled by decelerating. (c) The optical motions depend on the neighbor's eccentricity ( $\beta$, black curve), illustrated by a neighbor at a distance of $1 \mathrm{~m}$ slowing down by $-0.1 \mathrm{~m} / \mathrm{s}$. (d) In this case, the resulting angular velocity (blue curve) is a sine function of eccentricity, with a zero-crossing at $\beta=0^{\circ}$, a minimum at $\beta=-90^{\circ}$ (leftward motion), and a maximum at $\beta=90^{\circ}$ (rightward motion), whereas expansion rate (red curve) is a cosine function of eccentricity, with zero-crossings at $\beta=$ $\pm 90^{\circ}$ and a maximum at $\beta=0^{\circ}$ (expansion). If the neighbor speeds up, these curves flip about the $\mathrm{x}$-axis.

Collective motion. To formulate a visual model of collective motion, we substitute the visual control laws for local interactions (Equations 1 and 2) into a neighborhood function, which averages the influences of multiple neighbors. This yields,

$$
\ddot{\phi}_{p}=\frac{1}{n} \sum_{i=1}^{n} v_{i}\left[-c_{1}\left(\cos \beta_{i}\right) \dot{\psi}_{\iota}+c_{2}\left(\sin \beta_{i}\right) \dot{\theta}_{i}\right]
$$

$$
\ddot{r}_{p}=\frac{1}{n} \sum_{i=1}^{n} v_{i}\left[-c_{3}\left(\sin \beta_{i}\right) \dot{\psi}_{i}-c_{4}\left(\cos \beta_{i}\right) \dot{\theta}_{i}\right]
$$

in which pedestrian p's heading and speed are controlled by canceling the mean angular velocity $(\dot{\psi})$ and rate of expansion $(\dot{\theta})$ of all visible neighbors $(n)$, where the contributions of these two variables trade off as opposite functions of eccentricity $(\beta)$. The $180^{\circ}$ field of view is centered on the heading direction, as people tend to face in the direction they're walking (Grasso, Prevost, Ivanenko, \& Berthoz, 1998).

The effect of partial occlusion is incorporated by weighting each neighbor in proportion to their visibility (Dachner \& Warren, 2019), ranging from $v_{i}=0$ (fully occluded) to $v_{i}=1$ (fully visible). Visibility is set to 0 if its value falls below a threshold $\left(v_{t}=0.15\right)$, so $n$ is the number of visible neighbors above threshold. 
292

293

294

295

296

297

298

299

300

301

302

303

304

305

306

Importantly, due to the geometry of occlusion, the occluded region behind a near neighbor grows with distance, so the visibility of far neighbors tends to decrease with their separation in depth from near neighbors. Consequently, the range of the neighborhood varies with the crowd's opacity, the distance at which farther neighbors are completely occluded (Figure 1b). Note that visual occlusion invalidates the usual assumption of superposition, because the response to far neighbors is not in fact independent of the positions of near neighbors.

Basic properties of previous physical models fall out naturally from the visual model. First, canceling optical expansion yields collision avoidance without an explicit 'repulsion' force. Second, canceling optical contraction serves to maintain group cohesion without an explicit 'attraction' force. Third, canceling the combined angular velocity and expansion/contraction generates collective motion without an explicit 'alignment' rule. Critically, the fact that these variables obey the laws of optics explains the form of the neighborhood. Specifically, Euclid's law - the diminution of optical velocity with distance - accounts for the gradual decay of influence to the nearest (unoccluded) neighbors in a crowd, and the added effect of occlusion accounts for the more rapid decay within the crowd. The laws of optics thus explain the distance-dependent neighborhood of interaction, without an explicit decay function.

\section{Simulation results}

We tested the visual model by predicting the human trajectories in our virtual crowd experiments and comparing the results to our previous behavioral model (Equations S1-S3). In addition, to assess the contribution of visual occlusion to human responses, we compared the visual model to a motion model that was identical, except that the effect of occlusion was removed so responses were based on the optical motion variables alone (see SI for details). We find that the visual model outperforms the behavioral model and generalizes to real crowd data. It also outperforms the motion model, confirming the essential role of visual occlusion.

To simulate each experimental trial, the models were initialized with the participant's position, heading, and speed $2 \mathrm{~s}$ before the perturbation. For the behavioral model, the input on each time step was the position, heading, and speed of all virtual neighbors in a $90^{\circ}$ field of view. (If an experiment did not manipulate speed, we used the participant's recorded walking speed from each trial as input to the behavioral model.) For the visual model, the input was the angular velocity, expansion rate, eccentricity, and visibility of each neighbor, calculated from their positions relative to the model on each time step. The output of both models was the position, heading (and speed) of the simulated agent on the next time step, represented as time series for each trial. As a measure of model performance, we then computed the root mean squared error (RMSE) between each participant's mean time series in each condition and the corresponding mean time series for the model. To compare the two models, a Bayes Factor (BFvB) was used to estimate the relative strength of evidence.

Simulating two decay processes. First we simulated our double-decay experiment with the visual model. To compare it with the behavioral model, we needed to add a second exponential term to that model's decay function (Equation S4), which was estimated from the experimental data. The mean final heading for the two models is plotted in Figure $3 \mathrm{~b}$, together with the human results. Although both models are close to the $95 \%$ confidence intervals for the human data (shaded regions), the visual model (dotted curves) lies entirely within them.

Over the whole time series, the mean heading error for the visual model (RMSE $\left.=2.47^{\circ}\right)$ was significantly smaller than that for the behavioral model $\left(\mathrm{RMSE}=3.45^{\circ}\right)(t(9)=14.48, p<.001$, Cohen's $d=1.460)$, with decisive evidence in favor of the visual model $\left(B_{V B}>>100\right)$. The mean position error for the visual model $(\mathrm{RMSE}=0.241 \mathrm{~m})$ was also smaller than that for the behavioral model $(\mathrm{RMSE}=0.309 \mathrm{~m})(t(9)=8.46$, $p<.001$, Cohen's $d=0.294$ ), also decisive evidence for the visual model (BFvB $>>100)$. A similar comparison between the visual model and the motion model yielded decisive evidence for a contribution of occlusion (BFvM>100) (see Figure S1).

In sum, the visual model predicted the distance-dependent neighborhood more closely than the behavioral model, accounting for the two decay processes without an explicit decay function. The visual model thus grounds the neighborhood of interaction in the laws of optics. 
Re-simulating Rio, Dachner \& Warren (2018). As a further test of the models, we re-simulated the main experiment of Rio, Dachner, and Warren (2018), which perturbed heading or speed and manipulated the number and distance of perturbed neighbors (Figure 6a). Briefly, a participant $(n=10)$ walked with a virtual crowd of 12 neighbors, 5 in the near row (1.5m distance) and 7 in the far row ( $3.5 \mathrm{~m}$ distance). On each trial a subset of neighbors $\left(0,3,6,9\right.$, or 12 , predominantly in the near or far row) turned $10^{\circ}$ left or right, or changed speed by $\pm 0.3 \mathrm{~m} / \mathrm{s}$ from the base speed $(1.0 \mathrm{~m} / \mathrm{s})$. The participants' mean final heading and mean final speed appear in Figure $6 b, c$ (solid curves). Responses were larger when near neighbors were perturbed (blue) than when far neighbors were perturbed (red), indicating a decay of influence with distance.

Simulations of the visual model (dotted curves) and the behavioral model with the original single-decay function (dashed curves) are both close to the human data in Figure $6 \mathrm{~b}, \mathrm{c}$, falling within the $95 \%$ confidence intervals in nearly all conditions. The mean heading error was significantly smaller for the visual model than the behavioral model $\left(\mathrm{RMSEv}=1.97^{\circ}, \mathrm{RMSE}_{\mathrm{B}}=2.08^{\circ}\right),(t(9)=6.94, p<.001$, Cohen's $\left.d=0.871, B F_{v B}>100\right)$, although there were no differences for the mean speed error (RMSEv $=0.0627 \mathrm{~m} / \mathrm{s}$, $\left.\operatorname{RMSE}_{\mathrm{B}}=0.0640\right),\left(t(9)=1.15, p=0.281\right.$, Cohen's $\left.d=0.208, \mathrm{BF}_{01}=1.91\right)$, or the mean position error (RMSEv $\left.=0.193 \mathrm{~m}, \mathrm{RMSE}_{\mathrm{B}}=0.199 \mathrm{~m}\right),\left(t(9)=1.112, p=0.295\right.$, Cohen's $\left.d=0.082, \mathrm{BF}_{01}=1.96\right)$. Both models capture the human data quite well, although the evidence favors the visual model.

(a)

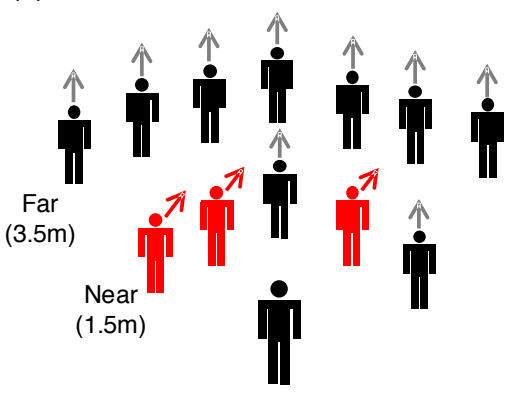

(b)

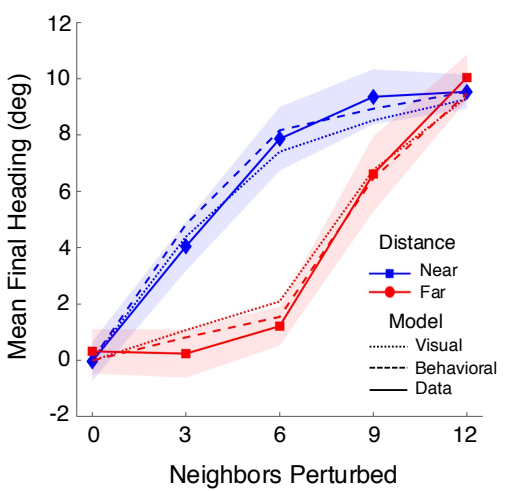

(c)

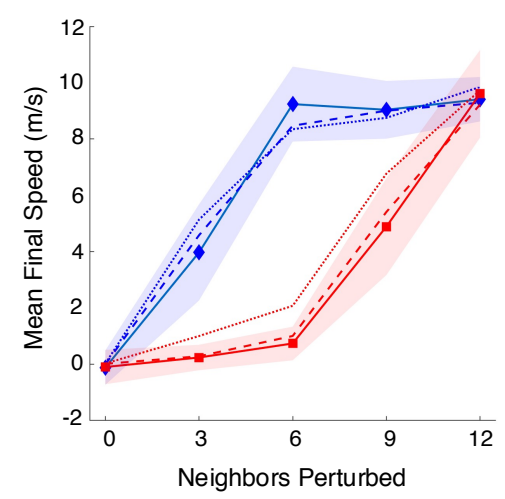

Figure 6. Re-simulation of Rio, Dachner \& Warren's (2018) experiment. (a) Schematic of virtual crowd $(n=12)$, illustrating a subset of neighbors whose heading is perturbed to the right. The number of perturbed neighbors in the near row $(1.5 \mathrm{~m})$ or the far row $(3.5 \mathrm{~m})$ was manipulated. Speed was also perturbed in a separate block of trials. (b) Results for heading perturbations: Mean final heading as a function of the number of perturbed neighbors; curves represent the primary perturbed row. (c) Results for speed perturbation: Mean final speed as a function of same. Solid curves correspond to human data, dotted curves to the visual model, dashed curves to the behavioral model with the single-decay function. Shaded regions represent $95 \%$ confidence intervals for the human data. [Panels $b$ and $c$ are modified from (8), with permission.]

The comparatively good performance of the behavioral model in this experiment stems from the fact that the single-decay function was originally fit to human swarms with a density similar to that of the virtual crowd, with nearest neighbors 1-3m distant. However, this empirical decay term does not generalize to larger distances in our double-decay experiment, whereas the visual model does, thanks to Euclid's law.

Do the observed RMSE values for the visual model indicate good performance? To benchmark the lower bound on model error, we estimated the inherent noise in the data due to gait oscillations and tracker error by computing the mean RMSE between a heading of $0^{\circ}$ and the mean time series for each participant in no-perturbation control trials. This yielded $R M S E_{L}=1.53^{\circ}$, revealing that the visual model $\left(\mathrm{RMSEv}_{\mathrm{f}}=1.97^{\circ}\right)$ is only $0.44^{\circ}$ from the best possible performance. Conversely, to estimate the upper 
bound on error - a failure to respond to the input - we computed the mean RMSE between a heading of $0^{\circ}$ and the mean time series for each participant on perturbation trials. This yielded RMSEu $=4.96^{\circ}$, indicating that the visual model $\left(\operatorname{RMSE}_{\mathrm{v}}=1.97^{\circ}\right)$ is much better than doing nothing. For speed, the visual model $\left(R_{M S E v}=0.0627 \mathrm{~m} / \mathrm{s}\right)$ is only $0.036 \mathrm{~m} / \mathrm{s}$ from the best possible performance $\left(R^{2} S_{L}=0.027 \mathrm{~m} / \mathrm{s}\right)$ and again is much better than doing nothing (RMSEu $=0.113 \mathrm{~m} / \mathrm{s})$.

In sum, the visual model accounts for Rio, et al.'s (2018) experiment as well or better than the behavioral model. Although the latter describes the relation between neighbor and participant velocities, the visual model explains that relation by appealing to the laws of optics: neighbors in the far row exert less influence because they have lower optical velocities due to Euclid's law and are partially occluded by near neighbors. We confirmed the role of occlusion by re-simulating the experiment with the motion model (without occlusion), yielding decisive evidence for the visual model (BF $\left.V_{M}>100\right)$ (Figure S2).

Human swarm. To test whether our findings for virtual crowds apply to real crowds, we recorded the walking trajectories of pedestrians in 'human swarms', intended to mimic collective motion in a public space. A group of participants was instructed to walk about a large hall, veering left and right, while staying together as a group. We then attempted to predict the trajectory of an individual participant from the movements of their neighbors, using the visual and behavioral models.

We recorded three different groups $(n=10,16,20)$ walking together in a large tracking area $(14 \mathrm{~m} \times 20 \mathrm{~m})$ for 2 min trials. Density was manipulated by varying the size of a starting box marked on the floor, and each group performed two trials at each density (measured as low $=1.72$ and high=2.10 participants $/ \mathrm{m}^{2}$ ), for a total of 12 trials. Head-mounted markers were tracked using 16 motion-capture cameras $(60 \mathrm{~Hz})$ and time series of position, heading and speed were computed as before. We then identified 10s segments of data in which $\geq 75 \%$ of the participants were continuously tracked, yielding 30 segments for analysis (17 high and 13 low density). In each segment, we simulated a focal participant at the back of the group and treated the tracked neighbors as input on each time step. We used the original single-decay function in the behavioral model (Equation S3), which had been fit to a sample of the swarm data.

Two segments of simulated swarm data appear in Figure 7. The heading time series (b) for the focal participant (red) is more closely captured by the visual model (blue) than the behavioral model (green) in both segments, whereas the speed time series (c) is better approximated by the behavioral model in Segment 1 and the visual model in Segment 10 (panel c). Over all 30 segments, the mean heading error was significantly lower for the visual model $\left(\mathrm{RMSE}_{\mathrm{v}}=15.0^{\circ}\right)$ than the behavioral model $\left(\mathrm{RMSE}_{\mathrm{B}}=22.9^{\circ}\right)(t$ $(29)=4.48, p<0.001$, Cohen's $d=0.806$ ), decisive evidence for the visual model (BF $\left.F_{B}>100\right)$, and so was the mean position error $\left(\operatorname{RMSE}_{v}=0.60 \mathrm{~m}, \mathrm{RMSE}_{B}=0.80 \mathrm{~m}\right)(t(29)=2.21, p<0.05$, Cohen's $d=0.338)$, anecdotal evidence $(B F v B=1.60)$. On the other hand, the mean speed error was significantly lower for the behavioral model $\left(\mathrm{RMSEv}_{\mathrm{v}}=0.224 \mathrm{~m} / \mathrm{s}, \mathrm{RMSE}_{\mathrm{B}}=0.146 \mathrm{~m} / \mathrm{s}\right)(t(29)=6.83 p<0.001$, Cohen's $d=1.198)$, decisive evidence $\left(\mathrm{BF}_{\mathrm{BV}}>>100\right)$, although variation in speed accounted for less of the total variation in position. Finally, the visual model performed better than the motion model (without occlusion) (BFvm $>40$ or better), confirming the importance of occlusion (Figure S3).

Overall, the visual model accounts for individual trajectories in real crowd data better than the behavioral model, even though the latter's decay term was fit to a subset of the same data. We attribute this advantage primarily to the effect of visual occlusion. Whereas the behavioral model approximates the decay with distance using a fixed exponential decay function, the visual model incorporates the dynamic occlusion that is visible on each trial, and is thus sensitive to the variation in occlusion over time. 
(a)
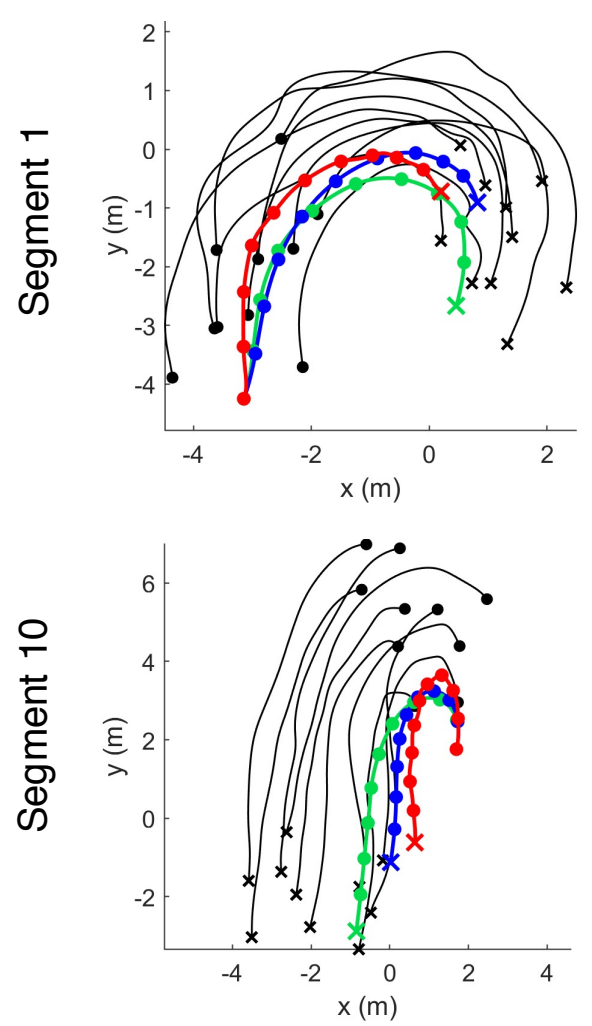

(b)
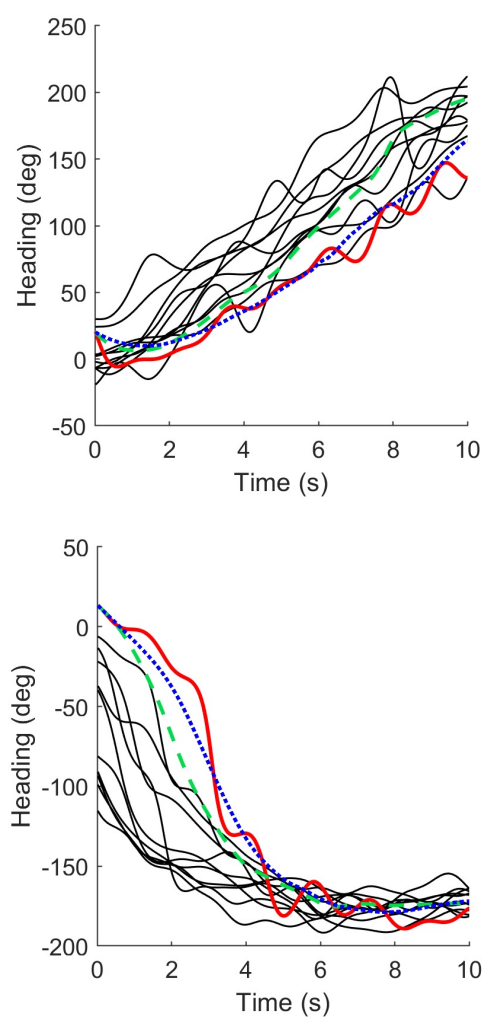

(c)
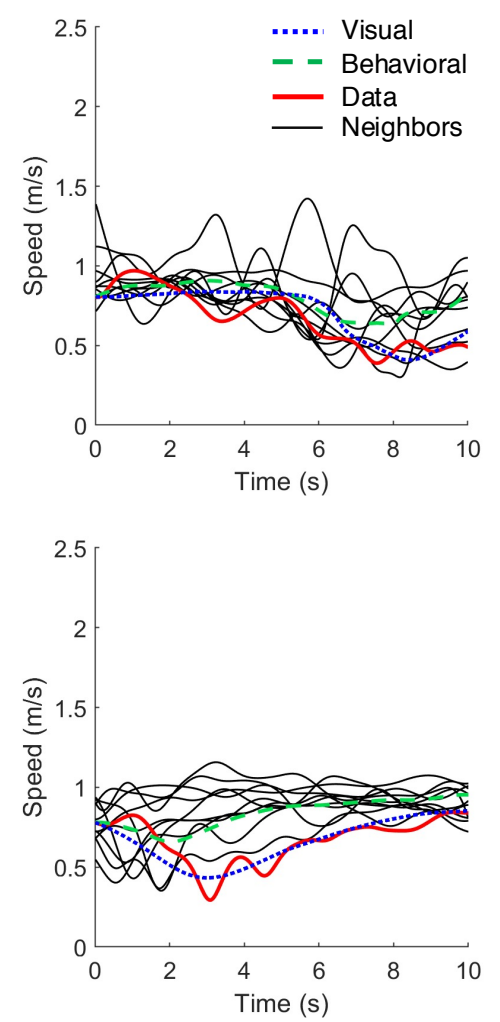

423

424

425

426

427

428

429

430

431

432

433

434

Figure 7. Sample data from the human swarm. Each row represents a 10 s segment of data, with the focal participant (red) and simulations of the visual model (blue) and the behavioral model (green). (a) Traces of position over time (Segment 1: $\mathrm{RMSE}_{V}=0.379 \mathrm{~m}, \mathrm{RMSE}_{\mathrm{B}}=0.818$; Segment 10: $\mathrm{RMSE}_{\mathrm{v}}=0.275 \mathrm{~m}, \mathrm{RMSE}_{\mathrm{B}}=1.389 \mathrm{~m}$ ). Thin gray curves $=$ tracked neighbors that were input to the model; $o=$ starting positions, $x=$ final positions, dots at $1 \mathrm{~s}$ intervals. (b) Time series of heading (Segment 1: RMSEv $=10.67^{\circ}, \mathrm{RMSE}_{B}=32.88^{\circ}$; Segment 10: RMSEv $=11.81^{\circ}$,

$\operatorname{RMSE}_{B}=23.62^{\circ}$ ). (c) Time series of speed (Segment 1: RMSE $=0.187 \mathrm{~m} / \mathrm{s}, R{ }^{2} E_{B}=0.162 \mathrm{~m} / \mathrm{s}$; Segment 10: RMSEv $=0.157 \mathrm{~m} / \mathrm{s}, R^{2} \mathrm{RSE}_{\mathrm{B}}=0.178 \mathrm{~m} / \mathrm{s}$ ). Note that errors in the swarm are higher than those for virtual crowds because they represent simulations of single trials rather than subject mean time series, and thus reflect gait oscillations and tracking errors.

\section{Discussion}

Nearly all models of collective motion in humans and animals attribute local interactions to hypothesized rules or metaphorical forces, described from an overhead view. In this article we developed a bottom-up visual model of human 'flocking', grounded in the optical information known to govern pedestrian interactions from an embedded viewpoint. In contrast to previous descriptive models, the visual model explains basic properties of interaction as a natural consequence of the laws of optics.

First, rules and forces of interaction are reduced to optical variables that control an individual's heading and speed. Instead of explicit 'repulsion' and 'attraction' forces, canceling optical expansion results in collision avoidance, while canceling optical contraction maintains group cohesion. Instead of an explicit 'alignment' rule, collective motion emerges from canceling the combined expansion/contraction and angular velocity of neighbors. This visual coupling yields a robust response regardless of the individual's position within the group. 
Second, the neighborhood of interaction is explained by the laws of optics, without an explicit distance term. The gradual decay to the nearest (unoccluded) neighbors in a crowd follows from Euclid's law, the diminution of optical velocity with distance. The more rapid decay within a crowd follows from the added effect of visual occlusion, which grows with the separation in depth between near and far neighbors. Consequently, the neighborhood range and number of neighbors $n$ do not depend on a fixed metric or topological distance (Ballerini et al., 2008; Strandburg-Peshkin et al., 2013), but vary with crowd opacity.

Interestingly, the visual model predicts that the effective neighborhood depends on crowd density, which we have confirmed in other experiments (Wirth, Dachner, Rio, \& Warren, 2021; Wirth \& Warren, 2018). In dense human crowds (1-2m apart), complete opacity can occur by $5 \mathrm{~m}$, whereas lower densities yield larger ranges of interaction. In the limit, starlings appear to adjust flock density to maintain a 'marginal opacity' at which individual birds can see through the entire flock(Pearce, Miller, Rowlands, \& Turner, 2014). The range of interaction might also be limited by a detection threshold for optical motion. However, adding a motion threshold to the visual model did not improve the fit to the data, perhaps because it was superseded by complete opacity.

Virtually all physical models assume the principle of superposition for combining binary interactions between individuals. However, the superposition principle is invalidated by the facts of visual occlusion. Because the influence of a far neighbor depends on the position of a near neighbor, an agent's response is not a linear combination of independent responses to each neighbor. While this may be computationally inconvenient, visual occlusion has large effects on local interactions and any model should take them into account.

Note that Euclid's law predicts an asymmetry in a pedestrian's response. Assume a neighbor at a given initial distance ahead: if they slow down, their distance decreases; whereas if they speed up by the same amount, their distance increases. Consequently, the corresponding rate of expansion is greater than the rate of contraction, respectively. This effect explains an asymmetric speed response we previously observed in pedestrian following (Bai \& Warren, 2019; Rio et al., 2014).

We found that the visual model generally outperforms the behavioral model, although they were quite similar in our re-simulation of Rio, Dachner \& Warren's (2018) data. This is attributable to the fact that the behavioral model approximates the effect of distance with an exponential decay term that was fit to human swarms that had a density similar to the virtual crowd display. However, this decay term did not generalize to larger crowd distances in our double-decay experiment, whereas the visual model did, with fixed parameters. The visual model thus explains the form of the neighborhood and generalizes to new conditions without re-parameterization.

We noted a limitation of the current visual model when simulating the swarm data. In five additional segments, the front of the crowd executed a $180^{\circ}$ hairpin turn and walked back toward the focal participant, generating a rapid expansion in the field of view. Whereas human participants kept walking forward, the visual model responded by slowing down and backing up to cancel the optical expansion. Similar but less extreme responses to U-turns may explain the higher speed RMSE for the visual model reported above. Clearly, the model needs to distinguish neighbors in a group from obstacles to be avoided, which may be as straightforward as distinguishing the face and back of other pedestrians.

Our findings suggest that characteristic patterns of collective motion in different species might result from reliance on different sensory variables. Humans cancel optical velocities, which will yield collective motion despite variation in the distance, density, and size of neighbors. In contrast, holding the visual angles of neighbors at a particular value would yield a school with a preferred spatial scale, whereas maintaining neighbors in particular visual directions would yield a flock with a preferred spatial structure (Ballerini et al., 2008).

In sum, we conclude that the local interactions underlying collective motion have a lawful basis in the visual coupling between neighbors. We are pursuing the consequences of this finding for other aspects of collective crowd motion, including information transfer, leadership networks (Lombardi, Warren, \& di Bernardo, 2020), and the role of visual attention (Lemasson, Anderson, \& Goodwin, 2009). 


\section{Methods}

\section{Experimental methods}

Human subjects: Participants were undergraduate and graduate students at Brown University, with normal or corrected-to-normal vision and none reported having a motor impairment. Different individuals participated in each experiment. A power analysis for repeated-measures ANOVA determined that to achieve a power of $1-\beta=0.85$ with an error probability of $\alpha=.05$ and an effect size of $0.5\left(\eta^{2}=0.2\right)$ required a sample size of 8 per experiment. The research protocol was approved by Brown University's Institutional Review Board, in accordance with the principles expressed in the Declaration of Helsinki. Informed consent was obtained from all participants, who were paid for their participation.

\section{Equipment: VR experiments were conducted in the Virtual Environment Navigation Lab (VENLab) at} Brown University. Participants walked freely in a $12 \mathrm{~m} \times 14 \mathrm{~m}$ tracking area while viewing a virtual environment in a wireless stereoscopic head-mounted display (HMD) (Rift DK1, Oculus, Irvine CA; $90^{\circ} \mathrm{H} x$ $65^{\circ} \mathrm{V}$ field of view, $640 \times 800$ pixels per eye, $60 \mathrm{~Hz}$ refresh rate). Head position and orientation were recorded with a hybrid inertial/ultrasonic tracking system (IS-900, Intersense, Billerica MA; $60 \mathrm{~Hz}$ sampling rate), and used to update the display with a total latency of 50-67 ms.

VR displays: The virtual environment was generated in Vizard software (WorldViz, Santa Barbara, CA). A green start pole and a gray orienting pole (radius $0.2 \mathrm{~m}$, height $3 \mathrm{~m}$ ) appeared $12.73 \mathrm{~m}$ apart on a ground plane with a grayscale granite texture and a blue sky. Virtual humans (WorldViz Complete Characters) were animated $3 D$ models $(M=8073 \pm 780$ polygons, each polygon with $2048 \times 2048$ pixels). They were initially placed on arcs with the participant's start pole at the center, at equally spaced eccentricities $\left( \pm 6^{\circ}\right.$, $\pm 19^{\circ}, \pm 32^{\circ}$, or $\pm 45^{\circ}$ ) from the participant's initial walking direction toward the orientation pole. These initial positions were then randomly jittered in polar coordinates, within an angular range of $5^{\circ}$ and a radial range of $0.2 \mathrm{~m}$. A different crowd configuration was generated for each trial. The virtual humans were animated with a walking gait with randomly varied phase (updated at $60 \mathrm{~Hz}$ ), and the crowd contained equal numbers of men and women and diverse races and ethnicities. During a trial, all virtual humans accelerated from a standstill $(0 \mathrm{~m} / \mathrm{s})$ to a speed of $1.0 \mathrm{~m} / \mathrm{s}$ on a straight path over a period of $3 \mathrm{~s}$, following a sigmoidal function (cumulative normal, $\mu=0, \sigma=0.5 \mathrm{~s}$ ) fit to previous human data. They continued to travel with the same heading and speed for an additional $2 \mathrm{~s}$. Then the heading of some or all virtual humans was perturbed by changing their walking direction over a period of $0.5 \mathrm{~s}$ following a similar sigmoidal function; the display continued for another $7 \mathrm{~s}$.

Procedure: Participants were instructed to "walk with the group of virtual humans" and to "treat them as if they were real people." On each trial, the participant walked to the green start pole and faced the gray orienting pole. After $2 \mathrm{~s}$, the poles disappeared and the virtual crowd appeared; $1 \mathrm{~s}$ later, a verbal command ("Begin") was played over the HMD's headphones and the crowd began walking. The display continued until the participant had walked for 12s, and a verbal command ('End') signaled the end of the trial. The start pole for the next trial then appeared nearby. Test trials were preceded by two practice trials to familiarize the participant with walking in the virtual environment.

Data processing: Data were processed in Matlab (Mathworks, Natick, MA). For each trial, the time series of head position in the horizontal $(X-Y)$ plane were filtered using a forward and backward fourth-order low-pass Butterworth filter to reduce tracker error and oscillations due to the step cycle. Time series of heading direction and walking speed were then computed from the filtered position data. A $0.6 \mathrm{~Hz}$ cut-off was used before computing heading to reduce lateral oscillations on each stride, while a $1.0 \mathrm{~Hz}$ cutoff was used before computing speed to reduce anterior-posterior oscillations on each step. The dependent measure was the final heading, the average heading direction during the last two seconds of each trial. Final heading responses that were more than 3 standard deviations away from that individual subject's mean were removed from the data set, then the mean final heading was computed for each participant in each condition.

Statistical analysis: Statistics were computed in Excel (Microsoft) and JASP (https://jasp-stats.org/). A preliminary 3-way Repeated-Measures ANOVA on final heading (Factor A x Factor B x left/right/control) was performed to confirm that the heading manipulation was successful in both directions. Left/right trials 
were then collapsed for further analysis by multiplying the final heading on left trials by -1 . The collapsed data were analyzed using 2-Way Repeated Measures ANOVA, followed by simple-effects tests or Bonferroni-corrected post-hoc $t$-tests (two-tailed). Effect sizes for ANOVAs were estimated using general eta-squared $\left(\eta_{G}^{2}\right)$; effect sizes for paired $t$-tests were estimated using Cohen's $d$, conservatively computed as the difference of the two means divided by the pooled variance of the two SDs (Dunlap, Cortina, Vaslow, \& Burke, 1996).

\section{First experiment: Decay to nearest neighbors}

Participants: Twelve participants (7 female, 5 male).

Displays: The crowd consisted of 2,4 , or 8 virtual humans, initially placed on one arc (radius $=1.8,3.0$, $4.0,6.0$ or $8.0 \mathrm{~m})$, at randomly assigned eccentricities $\left( \pm 6^{\circ}, \pm 19^{\circ}, \pm 32^{\circ}\right.$, or $\left.\pm 45^{\circ}\right)$. The heading direction of all virtual humans was perturbed to the right $\left(+10^{\circ}\right)$ on one-third of the trials, and to the left $\left(-10^{\circ}\right)$ on onethird of the trials; on the remaining control trials, they did not turn but continued walking on a straight path $\left(0^{\circ}\right)$.

Design: 5 crowd distances $(1.8,3.0,4.0,6.0,8.0 \mathrm{~m}) \times 3$ crowd sizes $(2,4,8$ neighbors $)$ yielded 15 conditions. Each participant received 4 heading perturbation trials $\left( \pm 10^{\circ}\right)$ and 2 control trials $\left(0^{\circ}\right)$ per condition, for a total of 90 trials. They were presented in a randomized order in a 1-hour session.

\section{Second experiment: Double-decay hypothesis}

Participants: Ten participants (6 female, 4 male) successfully completed Experiment 2; two additional participants discontinued the experiment due to discomfort.

Displays: The crowd consisted of 12 virtual humans, with four of them initially positioned on three concentric arcs (near, middle, far rows), with the start pole at the center. The arcs were separated by $2 \mathrm{~m}$, and radius of the near arc was varied (crowd distance $=2,4$, or $6 \mathrm{~m}$ ). The heading direction of all four neighbors in one row was perturbed to the right $\left(+10^{\circ}\right)$ on one-third of the trials, and to the left $\left(-10^{\circ}\right)$ on one-third of the trials; on the remaining one-third of trials (control trials), there was no perturbation $\left(0^{\circ}\right)$ and all neighbors walked on a straight path.

Design: 3 crowd distances $(2,4$, or $6 \mathrm{~m}) \times 3$ perturbed rows (near, middle, far) yielded 9 conditions. Each participant received 6 heading perturbation trials $\left( \pm 10^{\circ}\right)$ and 3 control trials $\left(0^{\circ}\right)$ per condition, for a total of 81 trials, presented in a randomized order in a 1-hour session.

Note that heading responses were expected to be smaller than in the first experiment because only onethird of the crowd was perturbed on each trial.

\section{Human swarm}

Participants. Three different groups (N=10 (5F, 5M), N=16 (6F,10M), N=20 (10F,10M)) participated in separate sessions as part of a larger study.

Equipment. Head position was recorded in a large hall with a Qualisys Oqus 16-camera infrared motion capture system (Qualisys, Buffalo Grove, IL) at $60 \mathrm{~Hz}$. The tracking area $(14 \mathrm{~m} \times 20 \mathrm{~m})$ and starting boxes of various sizes were marked on the floor with colored tape. Each participant wore a bicycle helmet with a unique constellation of five reflective markers on $30-40 \mathrm{~cm}$ stalks.

Procedure. Participants were instructed to walk about the tracking area at a normal speed for periods of 2 min, veering randomly left and right, while staying together as a group. Each group received two trials in each of two density conditions (low, high), determined by the size of the starting box. Participants began each trial in shuffled positions in a specified box; at a verbal 'go' signal, they began walking for 2 min, until a 'stop' signal. This yielded mean densities of 1.72 participants $/ \mathrm{m}^{2}$ in the low density condition and 2.10 participants $/ \mathrm{m}^{2}$ in the high condition, as measured within the bounding polygon of the crowd over all data (using the Matlab boundary and polyarea functions). 
591

592

593

594

595

596

597

598

599

600

601

602

603

604

605

606

607

608

609

610

611

612

613

614

615

616

617

618

619

620

621

622

623

624

625

626

627

628

629

630

631

632

633

634

635

636

637

638

639

Design. 3 groups $(\mathrm{N}=10,16,20) \times 2$ densities (low, high) with 2 trials in each condition, yielding a total of 12 trials with 24 min of raw data.

Data processing. The 3D positions of the markers on each helmet were reconstructed and tracked using QTM software (Qualisys), and the centroid for each helmet was computed using a custom algorithm. Due to infrared reflections in the hall, there were many tracking errors: $100 \%$ of the helmets were recovered in only $45 \%$ of all frames. The time series of head position in the horizontal $(x, y)$ plane was filtered in Matlab as before, and the heading direction and speed of each helmet was computed on each time step in which it was successfully tracked. We identified 3510 s segments of data in which $\geq 75 \%$ of the participants were continuously tracked. Five of these included hair-pin turns in which the front of the crowd walked back toward the focal participant, creating large optical expansions that caused the model to stop or back up; these were treated separately. This yielded 30 segments (17 high density and 13 low density) in which the positions of most neighbors were known. Rio, Dachner \& Warren (2018) previously simulated the same segments with the behavioral model, but subsequent improvements in data processing yielded more tracked neighbors, so the present results are more accurate.

\section{Simulation methods}

Simulations of virtual crowd experiments. Individual trials were simulated in Matlab using the Runge-Kutta method (ode45 function). The participant's position, heading, and speed $2 \mathrm{~s}$ before the perturbation were taken as the initial conditions. For the behavioral model (Equations S1-S2), the input on each time step was the position, velocity, and speed of the virtual humans in the participant's field of view for that trial. The double-decay experiment was simulated using the double-decay function (Equation S4) and Rio, Dachner \& Warren's experiment was simulated using the original single-decay function (Equation S3). Because speed control was not studied in the double-decay experiment, the virtual crowd's speed was constant, so the recorded time series of the participant's walking speed during each trial was treated as input to the behavioral model. For the visual model (Equations 3-4), the input was the angular velocity, optical expansion rate, eccentricity, and visibility of each virtual human, which were calculated from their positions relative to the simulated agent on each time step. The output of both models was the position, heading, and speed of the simulated agent on the next time step. This was represented as a time series of heading, a time series of speed, and time series of $(x, y)$ position for every trial.

Model comparisons. To compare the simulations with the human data, we calculated the model's mean time series for each participant in each condition, and then computed the root of the mean squared error (RMSE) between the model mean time series and the corresponding participant mean time series. The overall mean RMSE values for the two models were compared using paired $t$-tests (two-tailed) and scaled JZS Bayes Factors (BF) with a Cauchy prior of 0.707 , which indicate the relative strength of evidence for the hypotheses (Rouder, Speckman, Sun, Morey, \& Iverson, 2009). Note that the variability in final heading for the models is very small compared to the human data because gait oscillations and tracker error were not simulated; thus, the model means are compared with $95 \%$ confidence intervals for the human data in the figures.

Model performance benchmarks. The performance of any model is limited by the inherent noise in the human data due to gait oscillations and tracker error. To benchmark the lower bound on error, we estimated the fluctuations around a straight walking direction (heading $=0^{\circ}$ ) by computing the RMSEL between a value of $0^{\circ}$ and each participant's mean time series of heading on no-perturbation control trials. Conversely, to benchmark the upper bound on error, we estimated the error for a model that does not respond to the input (heading $=0^{\circ}$ ) by computing the RMSEu between a value of $0^{\circ}$ and each participant's mean heading time series on perturbation trials. These benchmarks indicate the range of model performance, from the best possible performance given the noise in the data, to the performance of a model that does nothing. (Of course, there exist even worse models that respond inappropriately.)

Human swarm simulations. The participant farthest back in the swarm at the beginning of each 10 s segment was selected as the focal participant. The focal participant's position, heading, and speed at the start of the segment were taken as initial conditions for the model simulation. For the behavioral model 
640 (Equations S1-S3), the position, heading, and speed of all tracked neighbors were treated as input to the 641 model, using the original single decay function. For the visual model (Equations 3-4), optical variables 642 (angular velocity, expansion rate, eccentricity, and visibility) were computed from the neighbor positions 643 relative to the simulated agent on each time step and used as input. Output was the position, heading, 644 and speed of the simulated agent on the next time step. This was represented as a time series of 645 heading, a time series of speed, and time series of $(x, y)$ position for each segment (e.g. Figure 7). The 646 RMSE between the time series for the simulated agent and the that for the focal participant was 647 computed for each segment, and the mean RMSE for each model was calculated over all 30 segments. 648 Note that the RMSE values were higher than those in the virtual crowd experiments because they 649 represented simulations of a single trial, rather than the mean time series of multiple trials; consequently, 650 they reflected larger fluctuations due to gait oscillations, tracker error, and incomplete tracking of 651 neighbors in the swarm.

\section{Acknowledgments}

653 This research was supported by the National Institutes of Health, R01EY010923 and R01EY029745 to 654 W.W., and T32 EY018080 to Brown University; the National Science Foundation, BCS-1431406 to W.W.; 655 and Link Foundation Fellowships to G.D. and T.W. Thanks to Adam Kiefer, Stephane Bonneaud, Michael 656 Fitzgerald, and the Sayles Swarm crew for their help during crowd data collection, and to Arturo

657 Cardenas, Eugy Han and the VENLab team for their assistance in processing and analyzing the datafiles.

\section{Author contributions}

659 G.D. and W.W. conceived the project and designed the research; T.W. and E.R. performed the 660 experiments; T.W. and W.W. analyzed the data; G.D. and W.W. developed the model; G.D. simulated the 661 data and analyzed the results; and G.D. and W.W. wrote the paper.

662

663

664

665

666

667 


\section{References}

Bai, J., \& Warren, W. H. (2019). The relative rate of optical expansion controls speed in 1D pedestrian following. Journal of Vision, 19(10), 52.

Bailo, R., Carrillo, J. A., \& Degond, P. (2018). Pedestrian models based on rational behaviour. In L. Gibelli \& N. Bellomo (Eds.), Crowd Dynamics, Volume 1: Modeling and simulation in science, engineering, and technology (Vol. I, pp. 259-292): Springer.

Ballerini, M., Cabibbo, N., Candelier, R., Cavagna, A., Cisbani, E., Giardina, I., . . Zdravkovic, V. (2008). Interaction ruling animal collective behavior depends on topological rather than metric distance: Evidence from a field study. Proceedings of the National Academy of Sciences, 105(4), 1232-1237.

Bastien, R., \& Romanczuk, P. (2020). A model of collective behavior based purely on vision. Science advances, 6(6), eaay0792.

Boltes, M., Zhang, J., Tordeux, A., Schadschneider, A., \& Seyfried, A. (2018). Empirical Results of Pedestrian and Evacuation Dynamics. In R. A. Meyers (Ed.), Encyclopedia of Complexity and Systems Science (pp. 1-29). Berlin, Heidelberg: Springer Berlin Heidelberg.

Calovi, D. S., Litchinko, A., Lecheval, V., Lopez, U., Escudero, A. P., Chaté, H., . . Theraulaz, G. (2018). Disentangling and modeling interactions in fish with burst-and-coast swimming reveal distinct alignment and attraction behaviors. PLoS Computational Biology, 14(1), e1005933.

Campanella, M., Hoogendoorn, S., \& Daamen, W. (2009). Improving the Nomad microscopic walker model. IFAC Proceedings Volumes, 42(15), 12-18.

Chen, X., Treiber, M., Kanagaraj, V., \& Li, H. (2018). Social force models for pedestrian traffic-state of the art. Transport reviews, 38(5), 625-653.

Chraibi, M., Tordeux, A., Schadschneider, A., \& Seyfried, A. (2018). Modelling of pedestrian and evacuation dynamics. In R. A. Meyers (Ed.), Encyclopedia of complexity and systems science (pp. 122). Berlin, Heidelberg: Springer Berlin Heidelberg.

Couzin, I. D., \& Krause, J. (2003). Self-organization and collective behavior in vertebrates. Advances in the Study of Behavior, 32, 1-75.

Couzin, I. D., Krause, J., James, R., Ruxton, G. D., \& Franks, N. R. (2002). Collective memory and spatial sorting in animal groups. Journal of Theoretical Biology, 218, 1-11.

Cucker, F., \& Smale, S. (2007). Emergent behavior in flocks. IEEE Transactions on Automatic Control, $52(5), 852-862$.

Dachner, G., \& Warren, W. H. (2014). Behavioral dynamics of heading alignment in pedestrian following. Transportation Research Procedia, 2, 69-76.

Dachner, G., \& Warren, W. H. (2017). A vision-based model for the joint control of speed and heading in pedestrian following. Journal of Vision, 17(10), 716.

Dachner, G., \& Warren, W. H. (2019). Dynamic occlusion reduces the influence of neighbors in human crowds. Paper presented at the International Conference on Perception and Action, Groningen, The Netherlands.

Dunlap, W. P., Cortina, J. M., Vaslow, J. B., \& Burke, M. J. (1996). Meta-analysis of experiments with matched groups or repeated measures designs. Psychological methods, 1(2), 170-177.

Dutra, T. B., Marques, R., Cavalcante-Neto, J. B., Vidal, C. A., \& Pettré, J. (2017). Gradient-based steering for vision-based crowd simulation algorithms. Computer Graphics Forum, 36(2), 337-348.

Faul, F., Erdfelder, E., Lang, A.-G., \& Buchner, A. (2007). G* Power 3: A flexible statistical power analysis program for the social, behavioral, and biomedical sciences. Behavior research methods, 39(2), 175191.

Frost, B. J., \& Sun, H. (2004). The biological bases of time-to-collision computation. In H. Hecht \& G. J. P. Savelsbergh (Eds.), Time-to-Contact (pp. 13-37). Amsterdam: Elsevier.

Gautrais, J., Ginelli, F., Fournier, R., Blanco, S., Soria, M., Chaté, H., \& Theraulaz, G. (2012). Deciphering interactions in moving animal groups. PLoS Comput Biology, 8(9), e1002678.

Giardina, I. (2008). Collective behavior in animal groups: theoretical models and empirical studies. HFSP Journal, 2(4), 205-219.

Gibson, J. J. (1979). The ecological approach to visual perception. Boston: Houghton Mifflin.

Grasso, R., Prevost, P., Ivanenko, Y. P., \& Berthoz, A. (1998). Eye-head coordination for the steering of locomotion in humans: An anticipatory synergy. Neurosci Lett, 253(2), 115-118.

Grégoire, G., Chaté, H., \& Tu, Y. (2003). Moving and staying together without a leader. Physica D: Nonlinear Phenomena, 181(3-4), 157-170. 
Helbing, D., Buzna, L., Johansson, A., \& Werner, T. (2005). Self-organized pedestrian crowd dynamics: Experiments, simulations, and design solutions. Transportation science, 39(1), 1-24.

Helbing, D., Farkas, I., \& Vicsek, T. (2000). Simulating dynamical features of escape panic. Nature, 407, 487-490.

Helbing, D., \& Molnár, P. (1995). Social force model of pedestrian dynamics. Physical Review E, 51, $4282-4286$.

Helbing, D., Molnár, P., Farkas, I., \& Bolay, K. (2001). Self-organizing pedestrian movement. Environment and Planning B: Planning and Design, 28, 361-383.

Hoogendoorn, S., \& Bovy, P. H. L. (2003). Simulation of pedestrian flows by optimal control and differential games. Optimal control applications and methods, 24(3), 153-172.

Huth, A., \& Wissel, C. (1992). The simulation of the movement of fish schools. Journal of Theoretical Biology, 156, 365-385.

Karamouzas, I., Skinner, B., \& Guy, S. J. (2014). Universal power law governing pedestrian interactions. Physical review letters, 113(23), 238701.

Katz, Y., Tunstrøm, K., Ioannou, C. C., Huepe, C., \& Couzin, I. D. (2011). Inferring the structure and dynamics of interactions in schooling fish. Proceedings of the National Academy of Sciences, 108(46), 18720-18725.

Köster, G., Treml, F., \& Gödel, M. (2013). Avoiding numerical pitfalls in social force models. Physical Review E, 87(6), 063305.

Lemasson, B. H., Anderson, J. J., \& Goodwin, R. A. (2009). Collective motion in animal groups from a neurobiological perspective: the adaptive benefits of dynamic sensory loads and selective attention. Journal of Theoretical Biology, 261(4), 501-510.

Leonard, N. E., Shen, T., Nabet, B., Scardovi, L., Couzin, I. D., \& Levin, S. A. (2012). Decision versus compromise for animal groups in motion. Proc Natl Acad Sci U S A, 109(1), 227-232. doi:10.1073/pnas.1118318108

Lombardi, M., Warren, W. H., \& di Bernardo, M. (2020). Nonverbal leadership emergence in walking groups. Scientific reports, 10(1), 1-10.

Mogilner, A., Edelstein-Keshet, L., Bent, L., \& Spiros, A. (2003). Mutual interactions, potentials, and individual distance in social aggregation. Mathematical Biology, 47, 353-389. doi:10.1007/s00285003-0209-7

Moussaid, M., Helbing, D., Garnier, S., Johansson, A., Combe, M., \& Theraulaz, G. (2009). Experimental study of the behavioural mechanisms underlying self-organization in human crowds. Proceedings of the Royal Society B, 276(1668), 2755-2762. Retrieved from http://www.ncbi.nlm.nih.gov/pubmed/19439442

Moussaïd, M., Helbing, D., \& Theraulaz, G. (2011). How simple rules determine pedestrian behavior and crowd disasters. Proceedings of the National Academy of Sciences, 108(17), 6884-6888.

Ngai, K. M., Burkle, F. M., Hsu, A., \& Hsu, E. B. (2009). Human stampedes: a systematic review of historical and peer-reviewed sources. Disaster medicine and public health preparedness, 3(4), 191195.

Ondrej, J., Pettré, J., Olivier, A.-H., \& Donikian, S. (2010). A synthetic-vision based steering approach for crowd simulation. ACM Transactions on Graphics, 29(4), 123: 121-129.

Partridge, B. L., \& Pitcher, T. J. (1980). The sensory basis of fish schools: relative roles of lateral line and vision. Journal of Comparative Physiology, 135, 315-325.

Pearce, D. J. G., Miller, A. M., Rowlands, G., \& Turner, M. S. (2014). Role of projection in the control of bird flocks. Proceedings of the National Academy of Sciences, 111(29), 10422-10426.

Pelechano, N., Allbeck, J. M., \& Badler, N. I. (2007). Controlling individual agents in high-density crowd simulation. In Proceedings of the 2007 ACM SIGGRAPH/Eurographics Symposium on Computer Animation, Aug. 3-7, San Diego, CA (pp. 99-108). Aire-la-Ville, Switzerland: Eurographics Association.

Pepping, G. J., \& Grealy, M. L. (Eds.). (2007). Closing the gap: The scientific writings of David N. Lee. Mahwah, NJ: Erlbaum.

Pita, D., Collignon, B., Halloy, J., \& Fernández-Juricic, E. (2016). Collective behaviour in vertebrates: a sensory perspective. Royal Society open science, 3(11), 160377.

Reynolds, C. W. (1987). Flocks, herds, and schools: a distributed behavioral model. Computer Graphics, $21,25-34$. 
Rio, K. W., Dachner, G. C., \& Warren, W. H. (2018). Local interactions underlying collective motion in human crowds. Proceedings of the Royal Society B, 285(1878), 20180611. doi:doi:10.1098/rspb.2018.0611

Rio, K. W., Rhea, C., \& Warren, W. H. (2014). Follow the leader: Visual control of speed in pedestrian following. Journal of Vision, 14(2), 4:1-16.

Romanczuk, P., Couzin, I. D., \& Schimansky-Geier, L. (2009). Collective motion due to individual escape and pursuit response. Physical review letters, 102(1), 010602.

Rouder, J. N., Speckman, P. L., Sun, D., Morey, R. D., \& Iverson, G. (2009). Bayesian t tests for accepting and rejecting the null hypothesis. Psychonomic bulletin \& review, 16(2), 225-237.

Schellinck, J., \& White, T. (2011). A review of attraction and repulsion models of aggregation: Methods, findings and a discussion of model validation. Ecological Modeling, 222, 1897-1911.

Srinivasan, M. V. (1998). Insects as Gibsonian animals. Ecological Psychology, 10(3-4), 251-270.

Strandburg-Peshkin, A., Twomey, C. R., Bode, N. W. F., Kao, A. B., Katz, Y., loannou, C. C., . . Couzin, I. D. (2013). Visual sensory networks and effective information transfer in animal groups. Current Biology, 23(17), R709-R711.

Sumpter, D. J. T. (2010). Collective animal behavior. Princeton, NJ: Princeton University Press.

Sumpter, D. J. T., Mann, R. P., \& Perna, A. (2012). The modelling cycle for collective animal behaviour. Interface Focus, 2(6), 764-773.

Vicsek, T., Czirók, A., Ben-Jacob, E., Cohen, I., \& Shochet, O. (1995). Novel type of phase transition in a system of self-driven particles. Physics Review Letters, 75(6), 1226-1229.

Vicsek, T., \& Zafeiris, A. (2012). Collective motion. Physics Reports, 517, 71-140.

Wagner, H. (1982). Flow-field variables trigger landing in flies. Nature, 297(5862), 147-148.

Warren, W. H. (1998). Visually controlled locomotion: 40 years later. Ecological Psychology, 10, $177-219$.

Warren, W. H. (2006). The dynamics of perception and action. Psychological Review, 113, 358-389.

Warren, W. H., \& Dachner, G. C. (2018). Comparing simple-radius and doughnut models of collective crowd motion. Journal of Vision, 18(10), 1038.

Warren, W. H., \& Fajen, B. R. (2008). Behavioral dynamics of visually-guided locomotion. In A. Fuchs \& V. Jirsa (Eds.), Coordination: Neural, behavioral, and social dynamics (pp. 45-75). Heidelberg: Springer.

Warren, W. H., Kay, B. A., Zosh, W. D., Duchon, A. P., \& Sahuc, S. (2001). Optic flow is used to control human walking. Nature Neuroscience, 4, 213-216.

Weitz, S., Blanco, S., Fournier, R., Gautrais, J., Jost, C., \& Theraulaz, G. (2012). Modeling collective animal behavior with a cognitive perspective: A methodological framework. PLoS ONE 7(6), e38588.

Wirth, T. D., Dachner, G. C., Rio, K. W., \& Warren, W. H. (2021). The neighborhood of interaction in human crowds is neither metric nor topological, but visual. manuscript submitted for publication.

Wirth, T. D., \& Warren, W. H. (2018). Metric vs. topological models of collective motion in human crowds. Journal of Vision, 18(10), 1036.

Zienkiewicz, A., Barton, D. A., Porfiri, M., \& Di Bernardo, M. (2015). Data-driven stochastic modelling of zebrafish locomotion. Journal of Mathematical Biology, 71(5), 1081-1105. 